\title{
Genetic Variants of FOXP2 and KIAA0319/TTRAP/THEM2 Locus Are Associated with Altered Brain Activation in Distinct Language-Related Regions
}

\author{
Philippe Pinel, ${ }^{1,2,3,4}$ Fabien Fauchereau, ${ }^{5,6,7}$ Antonio Moreno, ${ }^{1,2,3,4}$ Alexis Barbot, ${ }^{2}$ Mark Lathrop, ${ }^{8}$ Diana Zelenika, ${ }^{8}$ \\ Denis Le Bihan, ${ }^{2}$ Jean-Baptiste Poline, ${ }^{2,9}$ Thomas Bourgeron, ${ }^{5,6,7}$ and Stanislas Dehaene ${ }^{1,2,3,4}$ \\ ${ }^{1}$ INSERM, U992, Cognitive Neuroimaging Unit, F-91191 Gif/Yvette, France, ${ }^{2}$ CEA, DSV/I2BM, NeuroSpin Center, F-91191 Gif/Yvette, France, ${ }^{3}$ Université \\ Paris-Sud, Cognitive Neuroimaging Unit, F-91191 Gif/Yvette, France, ${ }^{4}$ Collège de France, F-75005 Paris, France, ${ }^{5}$ Laboratory of Human Genetics and \\ Cognitive Functions, Institut Pasteur, 75015 Paris, France, ${ }^{6}$ Université Paris Diderot, Sorbonne Paris Cité, F-75205 Paris, France, ${ }^{7} \mathrm{CNRS}$, URA 2182 Gènes \\ Synapses et Cognition, 75015 Paris, France, ${ }^{8}$ Centre National de Génotypage, 91057 Evry, France, and ${ }^{9}$ INRIA, Parietal, F-91191 Gif/Yvette, France
}

Recent advances have been made in the genetics of two human communication skills: speaking and reading. Mutations of the FOXP2 gene cause a severe form of language impairment and orofacial dyspraxia, while single-nucleotide polymorphisms (SNPs) located within a KIAA0319/TTRAP/THEM2 gene cluster and affecting the KIAA0319 gene expression are associated with reading disability. Neuroimaging studies of clinical populations point to partially distinct cerebral bases for language and reading impairments. However, alteration of FOXP2 and KIAA0319/TTRAP/THEM2 polymorphisms on typically developed language networks has never been explored. Here, we genotyped and scanned 94 healthy subjects using fMRI during a reading task. We studied the correlation of genetic polymorphisms with interindividual variability in brain activation and functional asymmetry in frontal and temporal cortices. In FOXP2, SNPs rs6980093 and rs7799109 were associated with variations of activation in the left frontal cortex. In the KIAA0319/TTRAP/THEM2 locus, rs17243157 was associated with asymmetry in functional activation of the superior temporal sulcus (STS). Interestingly, healthy subjects bearing the KIAA0319/TTRAP/THEM2 variants previously identified as enhancing the risk of dyslexia showed a reduced left-hemispheric asymmetry of the STS. Our results confirm that both FOXP2 and KIAA0319/TTRAP/THEM2 genes play an important role in human language development, but probably through different cerebral pathways. The observed cortical effects mirror previous fMRI results in developmental language and reading disorders, and suggest that a continuum may exist between these pathologies and normal interindividual variability.

\section{Introduction}

Human language is under strong genetic influence, as indicated by familial studies of clinical populations affected by language impairment (LI) or by reading disability (dyslexia) (Pennington et al., 1991; DeFries, 1996; Stromswold, 2001). A candidate gene for LI was first evidenced in members of the KE family affected by a missense mutation in FOXP2 gene (chromosome 7q31) that

\footnotetext{
Received Nov. 14, 2010; revised Sept. 20, 2011; accepted 0ct. 7, 2011.

Author contributions: P.P., T.B., and S.D. designed research; P.P., F.F., A.M., M.L., D.Z., and T.B. performed research; A.M., A.B., M.L., D.Z., D.L.B., and J.-B.P. contributed unpublished reagents/analytic tools; P.P., F.F., J.-B.P., and S.D. analyzed data; P.P., F.F., T.B., and S.D. wrote the paper.

This work was supported by the Pasteur Institute, the Centre National de Génotypage, Evry, University Denis Diderot Paris 7, INSERM, CEA, CNRS, Collège de France, and Fondation FondaMentale. A.B. was funded by IMAGEN project FP6 and J.-B.P. was partly funded by IMAGEN project FP6. IMAGEN receives research funding from the European Community's Sixth Framework Programme (LSHM-CT-2007-037286). This paper reflects only the author's views and the IMAGEN Community is not liable for any use that may be made of the information contained therein. We thank the LBIOM team (L. Hertz-Pannier, V. Joly, S. Roger, K. Ripert, S. Desmidt, and G. Mediouni-Cloarec) and other NeuroSpin investigators for contributing to the fMRI/genetic database by sharing fMRI scanning time and participants with us. We also thank Christophe Lalanne for his advice in statistical analysis.

The authors declare no competing financial interests.

Correspondence should be addressed to Philippe Pinel, Cognitive Neuroimaging Unit U992, INSERM-CEA, CEA/ SAC/DSV/DRM/NeuroSpin Center, F-91191 Gif/Yvette cedex, France. E-mail: philippe.pinel@cea.fr.

DOI:10.1523/JNEUROSCI.5996-10.2012

Copyright $\odot 2012$ the authors $\quad 0270-6474 / 12 / 320817-09 \$ 15.00 / 0$
}

disrupts the DNA-binding site of the protein (Lai et al., 2001). They exhibited severe speech and language deficits as well as orofacial dyspraxia (Vargha-Khadem et al., 1995). Implication of FOXP2 in vocal communication in mice and songbird (Haesler et al., 2004; Shu et al., 2005) and evidence of an accelerated evolution specific to the human lineage (Enard et al., 2002) reinforced the interest for FOXP2 in relation to the evolution of human language. Since then, other point mutations were reported across FOXP2 (MacDermot et al., 2005; Fisher and Scharff, 2009) and recent studies suggest associations between FOXP2 singlenucleotide polymorphisms (SNPs) and speech sound disorders (Zhao et al., 2010), schizophrenia (Sanjuán et al., 2006; Tolosa et al., 2010) or language skills (Peter et al., 2011). Similarly, for developmental reading disabilities, linkage and association studies have pointed to several susceptibility loci (DYX1-9) (Caylak, 2007). Among them, a limited number of risk allelic variations within the KIAA0319/TTRAP/THEM2 genes cluster on chromosome $6 \mathrm{p} 22$ have been reliably replicated in independent samples (Francks et al., 2004; Cope et al., 2005; Luciano et al., 2007; Paracchini et al., 2008; Newbury et al., 2011). Functional studies of human cell-lines suggest that the risk haplotype for dyslexia which spans these genes alters 
reading abilities by down-regulating KIAA0319 expression (Paracchini et al., 2006).

However, no study attempted to test whether FOXP2 and KIAA0319/TTRAP/THEM2 polymorphisms are associated with detectable variations of brain activity in normal adults. To address this issue, we correlated FOXP2 and KIAA0319/TTRAP/ THEM2 polymorphisms with human fMRI activation maps acquired during written and spoken language comprehension tasks in 94 healthy adults. Considering our sample size, we focused our investigation on a priori regions of interest (ROI), both in the genome and in the cortex. For KIAA0319/TTRAP/THEM2, we investigated SNPs in strong linkage disequilibrium (LD) with those reported in the dyslexia literature, spanning a part of KIAA0319, the KIAA0319 promoter, TTRAP and the first THEM2 intron. For FOXP2, we explored all SNPs of exons 1-17, covering the entire coding region. In the cortex, three ROIs were defined according to neuroimaging studies of dyslexia and $\mathrm{KE}$ family. Two regions covered areas atypically activated during a covert language task in the affected KE family members (Liégeois et al., 2003): the left inferior frontal gyrus (IFG) and the precentral and postcentral gyri. The third region, in the left lateral temporal lobe, broadly covered the site of reduced activation in dyslexics (Paulesu et al., 2001; Silani et al., 2005; Maisog et al., 2008). We predicted that FOXP2 variants would correlates with activation during language processing in the frontal regions, while variants within KIAA0319/TTRAP/THEM2 locus would correlates with activation in the temporal region.

\section{Materials and Methods}

Subjects. The genetic and functional (fMRI) data used in this study come from a database of hundreds of subjects described in detail by Pinel et al. (2007). Joint genetic and fMRI data were available for 94 independent subjects, mostly young Caucasian highly educated ( $48 \%$ of men, mean age $=24.7$ years old, $98 \%$ with high school diploma,). All subjects were right-handed (assessed by the Edinburgh inventory), native French speakers and they considered themselves as normal readers. Some of them $(11 \%)$ reported attending speech therapy for less than one year during childhood for expression, pronunciation, orthographic or reading difficulties. However, examination of the scores from timed tests of reading (time to correctly read aloud as fast as possible a list of 20 words and 20 pseudowords, respectively) showed that subjects' performances were spread over a continuous distribution and that none could be considered as an outlier. All subjects gave their consent to enter the protocol.

MRI data. We used a 5-min-long functional localizer to isolate in a reliable way the individual correlates of covert sentence reading, speech listening, motor action and mental calculation (Pinel et al., 2007). Ten short sentences were presented via visual and 10 via auditory stimulation in a random-like order among other tasks (motor instruction and arithmetic problems). Twenty flashing checkerboards served as control for the reading task. Sentences were displayed as four successive screens (250 $\mathrm{ms}$ ) separated by $100 \mathrm{~ms}$ interval, and each composed of group of one to three words, resulting in $1.3 \mathrm{~s}$ of visual stimulation. Auditory stimuli were digitally recorded by a male speaker (resolution of 16 bits and sampling frequency of $22.05 \mathrm{kHz}$ ) and had a similar duration (1.2-1.7 s). A postscan debriefing ensured that subjects could read all sentences without difficulty.

Anatomical and fMRI data were acquired on two 3 Tesla scanners (74 subjects scanned on a Brucker 3T and 22 on a Siemens 3T) with a TR of $2400 \mathrm{~ms}$ and 34 slices of $4 \mathrm{~mm}$ thickness covering the whole brain. Images were preprocessed [realignment, normalization to the Montreal Neurological Institute (MNI) template, resampled voxel size to $3 \mathrm{~mm}, 5 \mathrm{~mm}$ smoothing] with SPM5 and analyzed according to the statistical parametric mapping (SPM) general linear model (hemodynamic response function plus its derivative). Activation analyses were based on the contrast between reading sentences versus viewing flashing checkerboards and speech listening versus rest. These tasks recruit large frontotemporal networks, strongly lateralized to the left hemisphere (Pinel and Dehaene, 2010). We computed individual asymmetry maps from activation maps by subtracting, voxel by voxel, the activation of the right hemisphere from the corresponding one in the left hemisphere, after normalizing the right hemisphere onto the left one.

Brain regions of interest. Frontal ROIs were defined on an anatomical basis using the WFU PickAtlas Tool (Maldjian et al., 2003), based on a prior neuroimaging study of KE family members in a covert language task (Liégeois et al., 2003). We only considered the brain regions reported in that study which passed a significance threshold of $p<0.05$ corrected for multiple comparisons across the brain volume. We therefore did not consider other reported anomalies, in particular subcortical areas, although these regions are important candidates for further research.

The IFG ROI was based on the union of the pars opercularis, triangularis and orbitalis regions (covering 1723 voxels). The superior frontal ROI was based on the union of precentral and postcentral gyri (2847 voxels). This choice was made to take into account the proximity of precentral location reported by Liégeois et al. (2003) with the central sulcus. Finally, the left lateral temporal ROI, was constructed from the union of three spheres (radius $12 \mathrm{~mm}$ ) centered at temporal-lobe foci where a significant difference of activation had been detected between individuals with dyslexia and controls during a reading task (Paulesu et al., 2001). The peaks were at coordinates $-54,-50,14(\mathrm{~mm})$ in the superior temporal gyrus, $-60,-56,0$ in the middle temporal gyrus and $-52,-60,-14$ in the inferior temporal gyrus. To match brain cortical structures, this ROI was further intersected with a statistical image of the subjects' gray matter (composed by voxels common to $50 \%$ or more of the subjects' individual segmented gray matter images), resulting in a 1430 voxels region.

Genetic material. Subjects' saliva was collected with the DNA collection kit (OG-250) from DNA Genotek. DNA was extracted according the manufacturer's instruction. An important aspect for increasing the quality of the high-throughput DNA genotyping was to collect DNA in a small volume of $200 \mu \mathrm{l}$ of TE10:1. DNA samples were transferred to the French Centre National de Génotypage for genotyping. Samples that passed DNA quality control were genotyped with Illumina Human $1 \mathrm{M}$ Duo BeadChips which contain 1,199,187 markers for SNPs analyses. Samples were processed according to the manufacturer protocol. Genotypes data were generated using Illumina's BeadStudio software. Only samples that had been successfully genotyped for $>98 \%$ of the SNP markers were retained. SNPs with call rates of $<98 \%$, or showing departure from Hardy-Weinberg equilibrium $\left(p<10^{-6}\right)$ were excluded. In our results, genomic position is given according to NCBI build 36 , dbSNP b126.

Genetic regions of interest. Within the KIAA0319/TTRAP/THEM2 locus we limited our investigation to the regions that have been repetitively found associated with dyslexia susceptibility and reading skills. It was defined by two haplotypes blocks characterized with Haploview 4.2 from our sample, which included SNPs where strong associations with reading-related measures were reported. The first region covers partially KIAA0319 gene and the KIAA0319/TTRAP intergenic region where strong associations with a reading score were reported, among others, for rs2235676 and rs9467247 within a dense cluster of associations (Francks et al., 2004), rs3212236 (Harold et al., 2006; Dennis et al., 2009; Newbury et al., 2011) and rs9461045 (Dennis et al., 2009). The second region covers the TTRAP and a part of THEM2 gene where associations were reported for rs2143340 (Francks et al., 2004; Cope et al., 2005; Luciano et al., 2007; Dennis et al., 2009), rs3756819 (Paracchini et al., 2008; Rice et al., 2009), and rs1061925 (Francks et al., 2004). Finally, recent significant associations with normal variation in reading and spelling ability have been found with a haplotype spanning KIAA0319 and TTRAP (Luciano et al., 2007; Paracchini et al., 2008). The complete list of SNPs included in our analysis was the following: rs3756821, rs9461045, rs707887, rs1047782, rs3087943, rs3181244, rs1129644, rs3212232, rs3212231, rs3756819, rs1061925, rs17243157, 3181227, rs2223588, rs6928074, rs9461049, rs926529, rs1885211 (in fact, some SNP variations were in perfect linkage disequilibrium, resulting in 15 genuine polymorphisms). In a second step, we aimed to test whether the genetic/fMRI associations that we found could be extended to the SNPs reported from the litera- 
ture. Because most of them were not present in the Illumina BeadChips, we performed imputation using MACH1 software (Li et al., 2010), with 100 iterations, with a sample of 195 healthy individuals of Caucasian ancestry, including the 94 individuals published in the present study. The reference panel was CEU individuals (European ancestry) of the 1000 Genomes Project (Durbin et al., 2010) downloaded from the MACH1 website (data generated in June 2010).

For the FOXP2 gene, loci of tentative SNP associations are more widespread. The three most reliable SNPs, reported in at least two independent cohorts are located from position $113.5 \mathrm{Mb}$ up to $114.0 \mathrm{Mb}$ : rs923875 and rs2396722 were first reported in a haplotype associated with schizophrenia (Sanjuán et al., 2006). Recently, rs923875 was also reported in association with behavioral word reading efficiency and finger motor sequencing tests (Peter et al., 2011) while rs2396722 was associated with speech sound disorders in a cohort of 150 patients (Zhao et al., 2010). The SNP rs17137124 has been associated with Specific Language Impairment (Rice et al., 2009) and with modulation of frontal degeneration in elderly subjects (Padovani et al., 2010). The other published SNP associations were located in the same range of positions. In a more exploratory approach, we therefore considered all the SNPs of FOXP2, based on the GenBank accession number AF337817 used by Lai et al. (2001) covering all exons 1-17: rs6942634, rs2894699, rs1476535, rs10255943, rs10486026, rs10261780, rs10262103, rs4727799, rs17312686, rs2106900, rs17312861, rs10249234, rs12113612, rs10266297, rs10279936, rs6980093, rs7784315, rs7799109, rs12532920, rs17137124, rs10269986, rs7812028, rs17137135, rs1229761, rs1229758, rs12705966, rs10230087, rs7782412, rs1456029, rs12670585, rs6966051, rs17213159, rs1378771, rs12705971, rs12705973, rs2396766, rs12671330 (24 nonidentical polymorphisms). Imputation was also performed on this chromosome, as described above.

Individual SNP association with $\mathrm{PMRI}$ activation. For each SNP, subjects were grouped according to their allelic status: homozygous for the major allele, heterozygous, or homozygous for the minor allele. In agreement with common neuroimaging standards, groups were considered only if they contained a minimum of 12 subjects, resulting in either two or three groups of subjects for each SNP. The fMRI activation maps and asymmetry maps were entered in SPM models with groups as the variables of interest and scanner as a covariate. Statistical mapping was restricted to a gray matter mask (bilateral for activation images and lefthemisphere only, without loss of generality, for asymmetry images).

The $F$ test across groups was used to identify voxels with a significant genetic effect for the given SNP. The search for significant voxels was restricted to the above-defined ROIs. Statistical significance was asserted by permutation tests: we compared the size of each voxel's $F$ test with the distribution of the maximum $F$ value observed in 10,000 random shuffles of the subjects within the corresponding ROI, and considered as significant the voxels with an observed $F$ value exceeding $95 \%$ of this random distribution. Thus, any voxel passing this criterion can be considered as significant at $p<0.05$, corrected for the size of the restricted brain volume that was searched.

After identifying the voxels significantly associated with a given SNP, we summarized the overall effect of a given SNP in a given ROI by summing, for all significant voxels in this ROI, the values of $-\log _{10}(p)$ where $p$ is the significance of the individual voxel. The outcome was an overall estimate of the effect size of the given SNP, combining both the number of significant voxels and their individual significance level into a single quantity (Fisher, 1950; Benson et al., 1999). The significance of this quantity was again estimated by permutation testing: we compared the observed effect size with the distribution of the maximal effect size observed in 10,000 random shuffles of the subjects. An effect was deemed significant at a corrected level if the effect size exceeded $95 \%$ of this distribution $(p<0.05)$.

To further correct for the total number of SNPs tested, we applied a multiple-testing correction that accounts for physical LD among SNPs (Gao et al., 2008, 2010). This was done using the SimpleM software, which estimates the effective number of tests $\left(M_{\text {eff }}\right)$ from a principal component analysis of the LD structures. $M_{\text {eff }}$ reported here corresponds to the eigenvalues that explain $99.5 \%$ of the SNPs variation in our genetic ROIs. In our analysis, the total $M_{\text {eff }}$ correction applied to each $p$-value equaled 26 (16 for FOXP2 and 10 for the KIAA0319/TTRAP/THEM2 locus). Additionally, we also report a more stringent Bonferroni correction that requests effect size to exceed $1-\alpha / n$ percent of the random distribution, where $\alpha=0.05$ and $n$ is the number of nonidentical SNPs explored ( 24 for FOXP2 and 15 for the KIAA0319/TTRAP/THEM2 locus, for a total Bonferroni correction $n=39$ ). This approach is more conservative given that it does not consider the high correlation of nearby SNPs.

\section{Results}

The activations evoked by the reading task covered the left IFG and precentral gyri, bilateral anterior and posterior middle and superior temporal gyri and left fusiform gyrus (see detailed description of the networks and its asymmetry by Pinel et al., 2007; Pinel and Dehaene, 2010). All of these areas presented a strong left hemispheric lateralization.

Five SNPs of FOXP2 (in intron 2 and 3) were significantly associated with reading activation in two frontal regions, while one SNPs of the KIAA0319/TTRAP/THEM2 locus, located within THEM2, was associated with the asymmetry of activation in the temporal lobe (Fig. 1). All these SNPs survived the $\mathrm{M}_{\text {eff }}$ correction, and only two FOXP2 SNPs did not survive the Bonferroni correction.

Figure $2 a$ shows the location of these effects for the three most significant SNPs: rs6980093 and rs7784315 in FOXP2 and rs17243157 in THEM2 (given that rs7784315 and rs17137135 in FOXP2 exhibit the same degree of significance, we only considered the first SNP in our analyses). Although we explored rather large cortical ROIs, the two FOXP2 SNPs correlated with fMRI activation only at two restricted cortical sites in the left IFG and the dorsal part of left precentral gyrus (PG). Even though our reading paradigm differs from the covert verb generation task used by Liégeois et al. (2003), both of these frontal sites fell close (respectively $6.7 \mathrm{~mm}$ and $13.7 \mathrm{~mm}$ ) to the $\mathrm{fMRI}$ activation anomalies previously identified in the KE family. Likewise, for the THEM2 SNP, the correlation with fMRI activation during reading was seen at a cortical site in the posterior superior temporal sulcus (pSTS) which corresponds tightly to the dominant site of functional and anatomical anomalies in dyslexia. To test whether these genetic effects were specific to our brain ROIs during the reading task, we expanded the analysis to the whole-brain level. Figure 3 shows the sum of all binarized SNPs' $F$ test maps along FOXP2 and the KIAA0319/TTRAP/THEM2 locus, thresholded with a cluster size correction to avoid removing smaller effects as might occur with a too stringent voxel-based correction. This showed no major additional brain effects of these SNPs, as the genetic effects remained primarily centered on the left IFG, PG, and $\mathrm{pSTS}$ within the reading networks activated by our paradigm.

To further validate the double dissociation between FOXP2 and KIAA0319/TTRAP/THEM2 locus, we tested whether the association of a given polymorphism remained significant once the effect of the others were controlled for. Thus, we performed two additional SPM analyses, either on fMRI activation or on asymmetry maps, using an additive model with three joint predictors corresponding to the alleles of the three isolated SNPs (85 subjects remained after exclusion of allelic subgroups with $<12$ subjects for a given SNP polymorphism). The association of FOXP2 SNP rs6980093 (AA > GG) with activation in the left IFG remained significant $\left(t_{85}=4.64\right.$, voxel $p=0.009$ corrected for the corresponding ROI). The association of FOXP2 SNP rs7784315 $(\mathrm{TC}>\mathrm{TT})$ with precentral activation was also significant $\left(t_{85}=5.03\right.$, voxel $p=0.003$ corrected for the corresponding ROI). Finally the association of the THEM2 SNP rs17243157 (CC > TC) with func- 

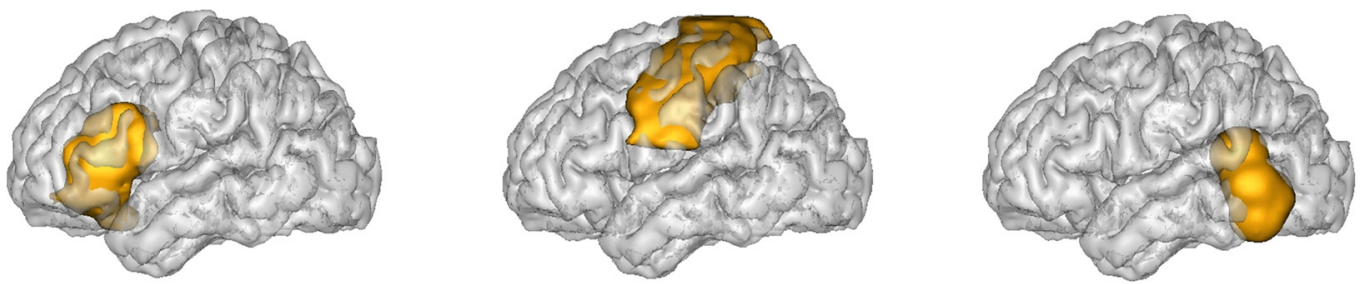

\begin{tabular}{cccc}
\multicolumn{4}{c}{ inferior frontal ROI } \\
\hline SNP & $\mathrm{P}_{\text {unc. }}$ & $\begin{array}{c}\mathrm{P}_{\text {corr. }} \\
\left(\mathrm{M}_{\text {ent }}\right)\end{array}$ & $\begin{array}{c}\mathrm{P}_{\text {corr. }} \\
\text { (Bonfer.) }\end{array}$ \\
\hline$\underline{\text { Left activation }}$ & & & \\
rs10249234 (A) & 0.0019 & 0.0494 & 0.0741 \\
rs6980093(A) & 0.0011 & 0.0286 & 0.0429
\end{tabular}

\begin{tabular}{cccc}
\multicolumn{4}{c}{ precentral ROI } \\
\hline SNP & $\mathrm{P}_{\text {wic. }}$ & $\begin{array}{c}\mathrm{P}_{\text {corr. }} \\
\left(\mathrm{M}_{\text {erf }}\right)\end{array}$ & $\begin{array}{c}\mathrm{P}_{\text {corr. }} \\
\text { (Bonfer.) }\end{array}$ \\
\hline$\underline{\text { Left activation }}$ & & & \\
$r s 7784315(\mathrm{C})$ & 0.0007 & 0.0182 & 0.0273 \\
$r s 7812028(\mathrm{~A})$ & 0.0021 & 0.0546 & 0.0819 \\
$r$ s $17137135(\mathrm{~A})$ & 0.0007 & 0.0182 & 0.0273 \\
Asymmetry & & &
\end{tabular}

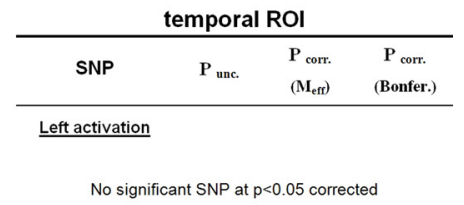

Asymmetry

No significant SNP at $p<0.05$ corrected

No significant SNP at $p<0.05$ corrected

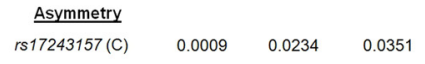

Figure 1. Significant association of SNPs within FOXP2 and KIAA0319/TTRAP/THEM2 locus with functional activation and asymmetry in frontal and temporal ROIs. The first row shows the three cerebral ROIs explored (yellow), plotted onto a transparent 3 D rendering of the left hemisphere (Anatomist/BrainVISA package). Statistical results are reported below. The uncorrected $p$-value $\left(p_{\text {unc. }}\right.$ ) corresponds to a single SNP effect. Only SNPs that achieved a correction by the effective number of tests $\left(M_{\text {eff }}\right)$ are reported ( $\left.p_{\text {corr. }}<0.05\right)$. Bonferroni correction is also reported (Bonfer.) for each SNP. Letters indicate the allele associated with the greater activation or asymmetry.

tional asymmetry was still significant in the temporal ROI $\left(t_{85}=\right.$ 4.43, voxel $p=0.017$ corrected for the corresponding ROI).

To better understand the fMRI/SNP associations, we examined how profiles of fMRI activation vary with allelic status at the relevant cortical peaks in both hemispheres, both during the reading task and during an independent speech listening task (Fig. 2b). FOXP2 rs6980093 polymorphism was associated with differences in IFG. Higher levels of activation where found associated with A allele, not only during the reading condition originally used to identify this peak $\left(F_{(2,90)}=12.89, p=1.2 \times 10^{-5}\right)$ but also during speech listening $\left(F_{(2,90)}=3.32, p=0.040\right)$, suggesting an effect on abstract supramodal language processes. Both effects were bilateral (no hemisphere $\times$ allele interaction). On the contrary, FOXP2 rs7784315 polymorphism was associated with variations of precentral activation during reading only $\left(F_{(1,91)}=19.76, p=2.4 \times 10^{-5}\right)$ with higher activation for TC compared with TT genotype. This significant left-lateralization (hemisphere $\times$ allele, $p=0.002$ ) and absence of effect in the listening condition are likely to reflect an association with more peripheral aspects of reading such as covert articulation. Finally, the THEM2 rs17243157 was strongly associated with asymmetry of the pSTS activation during reading (hemisphere $X$ allele interaction $F_{(2,90)}=20.07, p=2.2 \times 10^{-5}$, higher hemispheric difference for CC compared with TC genotype), with a significant effect on the left hemisphere $\left(F_{(1,90)}=10.59, p=0.002\right.$, higher activation for CC compared with TC genotype) during reading and a small effect in the same direction during speech listening $\left(F_{(1,90)}=3.33, p=0.036\right.$, one-tailed $)$. Both its cortical localization and its joint activation during reading and listening tasks are compatible with a contribution to phonological processing.

We then expanded our exploration of genetic associations with the frontal and temporal peaks to larger segments surrounding our original candidate genes. This analysis revealed that effects were restricted to the isolated SNPs and rarely passed a $-\log _{10}(p)$ value of 2 (Fig. $2 c$ ). Imputation allowed us to obtain a more detailed description of association within the KIAA0319/ TTRAP/THEM2 locus (Fig. 4) and to test candidate SNPs from the literature. SPM5 analyses were performed for imputed polymorphisms comprising the Illumina BeadChips' SNPs and variants from the 1000 Genomes Project (Durbin et al., 2010). At the temporal peak, associations were found with rs3181244, rs2143340, rs3756819, rs1061925 and rs17243157, which were previously associated with reading skills (Francks et al., 2004; Cope et al., 2005; Luciano et al., 2007; Paracchini et al., 2008; Dennis et al., 2009; Rice et al., 2009). The peak of association $\left(-\log _{10}(p)=5.11\right)$ was found at position 24777721 (labeled chr6:24777721 in the 1000 Genomes Project). After genotype correction by Sanger sequencing of this SNP (successful on 86 individual, 7\% of errors in imputed data), a high but lower significance was finally found $\left(-\log _{10}(p)=\right.$ 4.09 ) and reported in Figure 4 (first SNP right to rs17243157). Because several SNPs were in high LD, we could isolate a haplotype block $\left(r^{2}>0.44\right.$ in our larger European population of 195 subjects) that covers genes KIAA0319, TTRAP and THEM2. This analysis shows that the second most frequent haplotype (11.7\% of Europeans), includes a combination of alleles here associated in independent tests with a reduced pSTS asymmetry (Fig. 4). This allelic combination corresponds precisely to the risk alleles previously associated with dyslexia.

For the FOXP2 gene, similar examination of $\mathrm{fMRI} /$ genetic associations at the two frontal peaks around rs6980093 and rs7784315 reveal that they belong to two different haplotype blocks (Fig. 5). First, the SNP rs7784315, associated with the precentral activation, is in high LD with SNPs covering the alternatively spliced exon $3 \mathrm{~b}$, and nearby exons $3 \mathrm{a}$ and 4 , which are predicted to introduce stop and start codons, respectively (Fig. 5, top). Second, the SNP rs6980093 is in high LD with SNPs covering various exons (1-3a) and transcription start sites at exons 1 , $1 \mathrm{~b}$ and 2 (Fig. 5, bottom) with a maximal association peak around exons $2 \mathrm{~b}-3 \mathrm{a}$. The second haplotype block $\left(r^{2}>0.75\right)$ encompasses two genetic variants of FOXP2 recently reported as being associated with articulation scores (rs12533005) (Peter et al., 2011) and schizophrenia (rs2396753) (Sanjuán et al., 2006). The haplotype block formed by the three SNPs suggest that the first most frequent haplotype in the European population (49.9\%) is composed by risk alleles of rs12533005, rs2396753 and the A allele of rs6980093. Interestingly, rs6980093 is also in high LD (in our group of 195 subjects with European ancestry) with rs17137124 $\left(r^{2}=0.50\right)$ which have been reported to modulate frontal degeneration in elderly subjects (Padovani et al., 2010), 

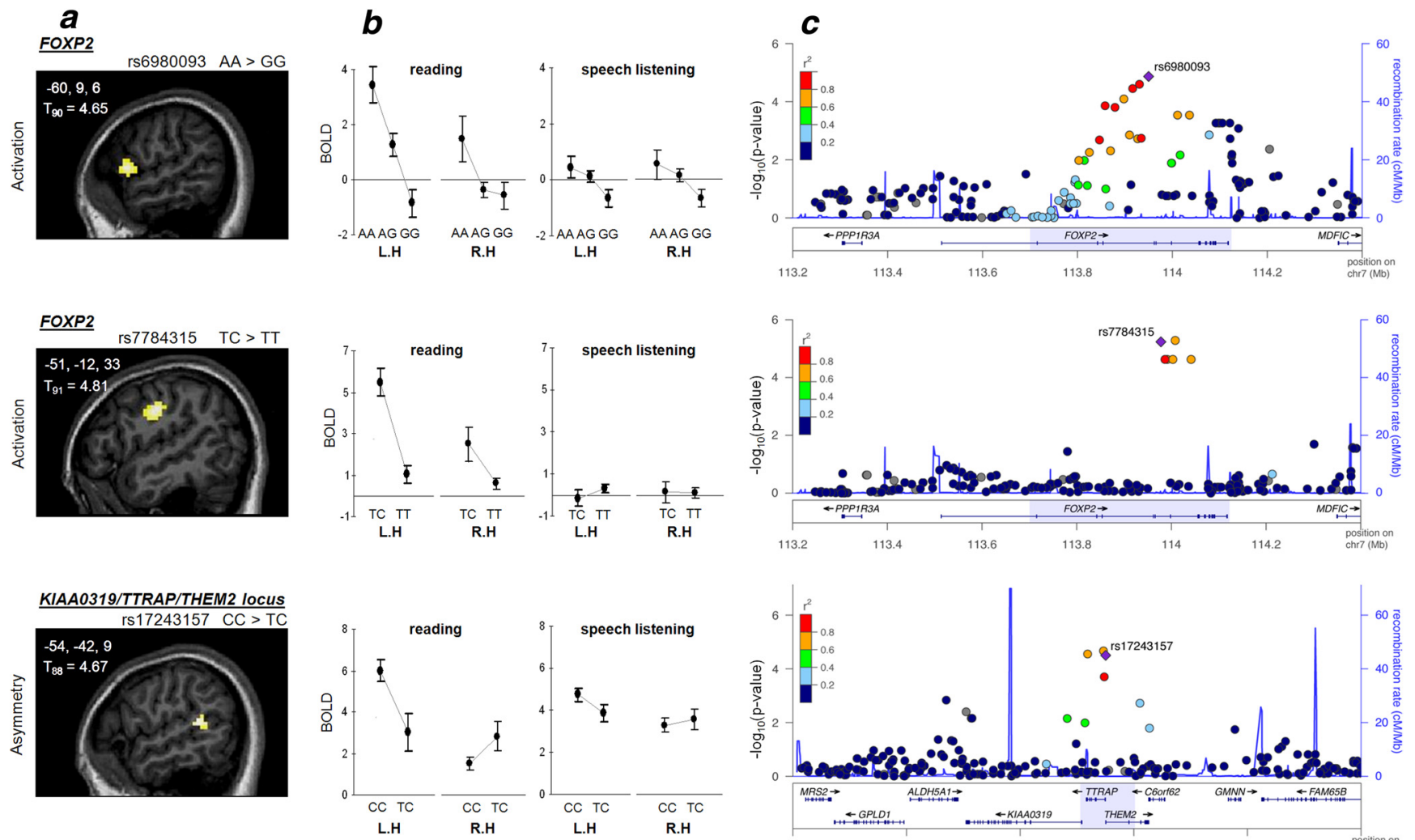

Figure 2. Association of SNPs within FOXP2 and KIAA0319/TTRAP/THEM2 locus with reading- and speech-related brain activation. $\boldsymbol{a}$, Brain regions showing a significant effect of SNP allelic variation through a whole-brain statistical analysis either on reading activation or on asymmetry maps (Student's $t$ test, voxel $p<0.001$, cluster-extent $p<0.05$ corrected) plotted on a sagittal anatomical slice. $\boldsymbol{b}$, Activation (percentage change in BOLD signal) at each cortical peak of the left hemisphere (L.H) and the symmetrical position in the right hemisphere (R.H), for reading and speech listening tasks, plotted separately for the different allelic groups. $c$, Variation in the strength $\left(-\log _{10}(p)\right.$, left $y$-axis) of the fMRI-SNP relation across the candidate genetic sites (blue background in the genes annotation window) and neighboring sites of the genome [performed with LocusZoom software (Pruim et al., 2010)]. The right axis shows the recombination rate estimated in the Hapmap phase II CEU population. The $x$-axis represents the SNP's physical position in megabases (Mb). Colored dots refer to the $r^{2}$ linkage disequilibrium value (see color scale), estimated between each SNP and the reference SNP (violet diamond) from the CEU panel (European ancestry).
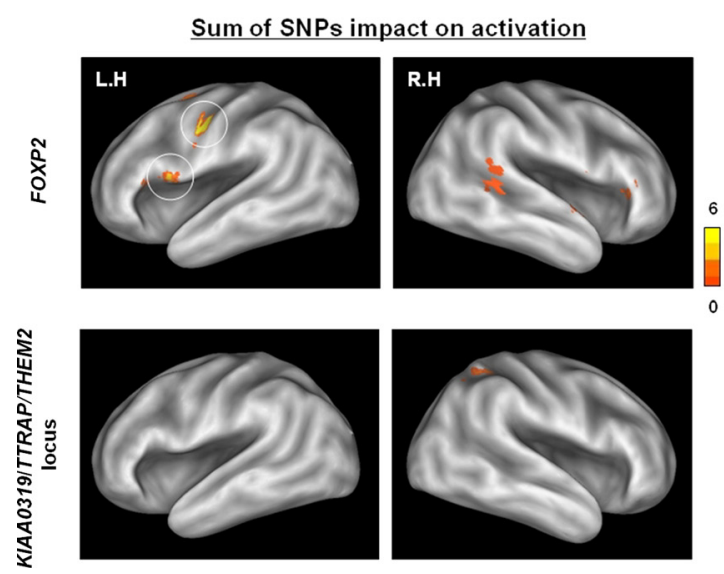

Figure 3. Whole-brain association of SNPs within FOXP2 and KIAA0319/TTRAP/THEM2 locus with activation and asymmetry maps. The figures show the sum of all binarized SNPs' $F$ test maps for individual SNPs $(p<0.05$ corrected for cluster extent for a voxelwise threshold of $p<0.001$ ), plotted on inflated hemispheres (Caret software).

and two other SNPs, rs10230558 and rs7782412, which have been associated with reduced score in word reading tasks (Peter et al., 2011), although these SNPs did not show any association with fMRI data in the present sample.

\section{Discussion}

Twin studies demonstrated that interindividual variability in language skills rests in part on a genetic basis (Stromswold, 2001).
Sum of SNPs impact on asymmetry
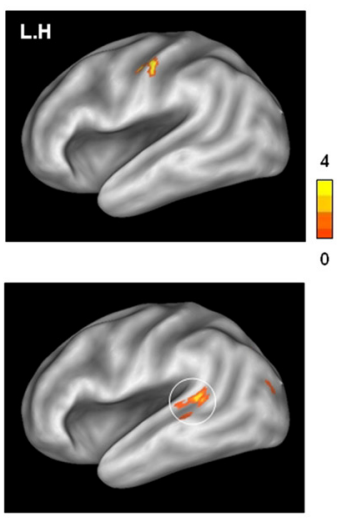
lateralization
We showed that a similar variability exists at the cortical level and can be partially related to distinct genetic sites, even within a single reading task. Our relatively small sample size prevented us to explore the entire genome. Instead, we selected two candidate genetic regions located in FOXP2 and in the upstream regions of KIAA0319 which are consistently involved in pathologies of language development and for which functional pathways have been proposed (Galaburda et al., 2006; Fisher and Scharff, 2009). We found two FOXP2 SNPs associated with bilateral inferior frontal activity (rs6980093) and with left precentral activity (rs7784315), respectively, and a SNP within the KIAA0319/TTRAP/THEM2 locus (rs17243157) associated with temporal functional asymmetry.

The KIAA0319/TTRAP/THEM2 locus and temporal

Association with the pSTS functional lateralization for language was found in a THEM2 SNP rs17243157 at a cortical peak that fits with cerebral anomalies described in dyslexia. Indeed, reduced superior temporal anatomical asymmetry is predictive of reading 


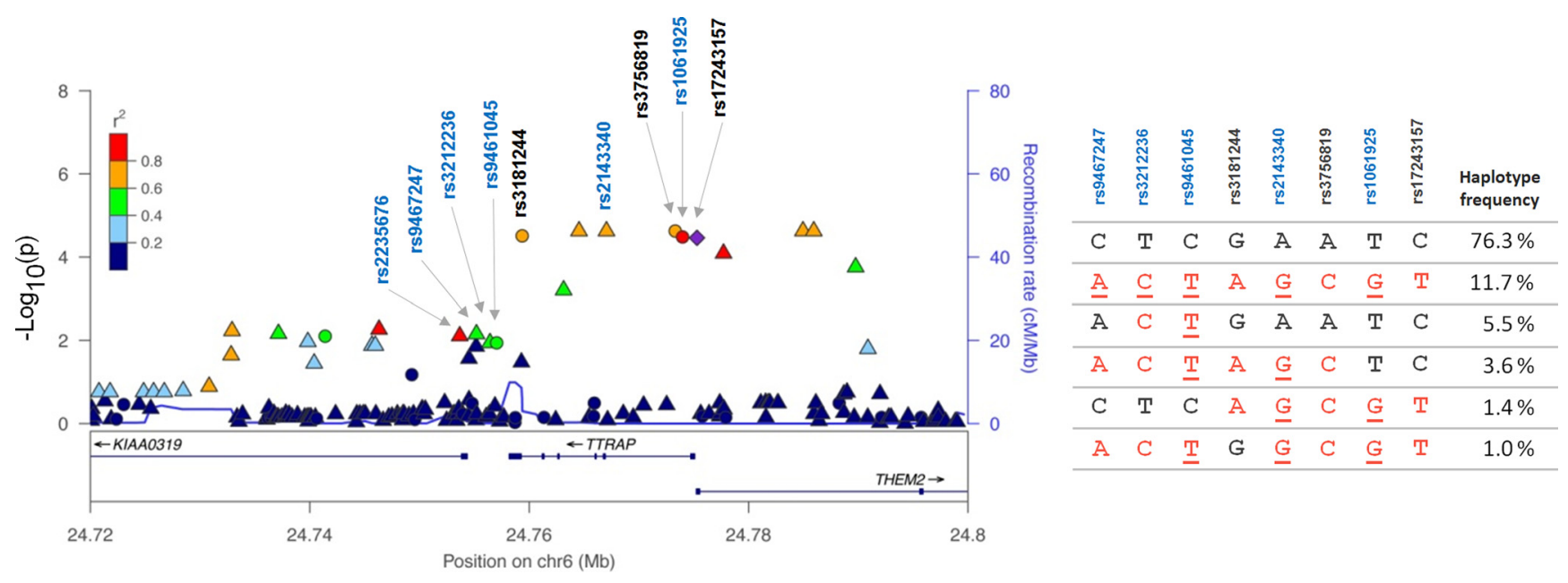

Figure 4. Convergence of the DYX2 genetic variants for lower asymmetry in the pSTS with the risk haplotype for dyslexia identified in previous studies (Francks et al., 2004; Cope et al., 2005; Harold et al., 2006; Luciano et al., 2007; Paracchini et al., 2008; Dennis et al., 2009; Rice et al., 2009; Newbury et al., 2011). On the left is displayed a zoomed view within the KIAA0319/TTRAP/THEM2 region of the observed genetic association with functional pSTS asymmetry. The significance of the association at the STS peak $\left(-\log _{10}(p)\right)$ is reported for the genotyped SNP (circles) as well as for the imputed SNPs (triangles). Black labels correspond to the SNPs showing an association with pSTS asymmetry at the voxel level isolated in our study, blue labels correspond to the candidate SNPs reported in a previous publication (Peter et al., 2011). Dots colors refer to the $r^{2}$ linkage disequilibrium value estimated between each SNP and the reference SNP (violet diamond). $r^{2}$ was estimated from data of the 1000 Genomes Project (June 2010 CEU; Durbin et al., 2010). Other annotations are described in Figure 2c. The table on the right shows the most frequent haplotypes corresponding to the labeled SNPs, ordered by their frequency. Underlined letters indicate alleles previously associated with a risk of dyslexia and red letter alleles associated with a lower pSTS asymmetry.

disability (Galaburda et al., 1985; Leonard et al., 2001) and a recent meta-analysis isolated the strongest functional anomalies in dyslexia within the left inferior and lateral temporal lobe (Maisog et al., 2008).

The observed SNP shares a common haplotype block with other variants of THEM2, TTRAP and KIAA0319, making difficult to dissociate their effects. Independent $\mathrm{AMRI} / \mathrm{SNP}$ association tests showed that alleles associated with lower STS asymmetry constitute a combination of variants that corresponds tightly to the risk alleles for dyslexia. It contains alleles that downregulates KIAA0319 expression, in particular the functional variant rs9461045 which creates a nuclear protein-binding site for transcription factor (Dennis et al., 2009). This downregulation affects neuronal migration (Paracchini et al., 2006), providing a putative biological mechanism for the anatomical anomalies found in the brain of dyslexic patients (Galaburda et al., 1985). Such a mechanism may also contribute to variations in functional pSTS asymmetry which may, in turn, relate to normal interindividual variations in reading and spelling abilities (Luciano et al., 2007; Paracchini et al., 2008). These results suggest that similar genetic variants may contribute to both normal interindividual variability and dyslexia, dyslexic subjects lying below an arbitrary cutoff of a continuous distribution of reading scores (Shaywitz et al., 1992).

Interestingly, the association with the KIAA0319/TTRAP/ THEM2 variants generalized to the speech listening task, suggesting that the effect is not unique to reading and might arise from a modulation of phonological processes. This finding fits with the reported correlation between underactivation of the superior temporal gyrus in dyslexics during speech listening, phonemic awareness scores, and atypical activation at this site during a sound/letter matching task (Blau et al., 2009). We tentatively hypothesize that KIAA0319 plays a role in the lateralization of phonemic processing in the pSTS. Since human newborns already present a left-hemispheric functional STS asymmetry for speech (Dehaene-Lambertz et al., 2002; Bernal et al., 2010), such a genetic influence is likely to occur early in life.
The FOXP2 gene and frontal language-related activation

FOXP2 associations were found with left frontal areas, nearby the cortical anomalies reported in the KE family (Liégeois et al., 2003). Activation profiles suggest that the IFG associated with FOXP2 polymorphism, close to Broca area, is involved in both language perception and production while the precentral site, which lies in a primary motor cortex region involved in lips and larynx motor representation (Brown et al., 2009), is likely related to articulation processes. Our findings may thus help to disentangle the coexistence of both linguistic and articulation deficits in these patients (Vargha-Khadem et al., 1995, 1998). A more common form of developmental LI termed Specific Language Impairment (SLI) has been associated to additional genetic loci (Bartlett et al., 2002; SLIC, 2004). The small number of functional neuroimaging studies and the heterogeneity of the patients groups make difficult to draw consensual conclusions about brain correlates of SLI. However, anomalies in the IFG were also reported in brain anatomy (Gauger et al., 1997; De Fossé et al., 2004; Leonard et al., 2006) and in event-related potential studies: grammatical-SLI patients showed an absence of early left anterior negativity during syntactic violations (Fonteneau and van der Lely, 2008) and SLI children presented an absence of early right anterior negativity during music syntax processing (Jentschke et al., 2008). Thus, IFG appears crucial in language pathologies and may be a promising candidate endophenotype in the search for new genetic associations.

Interestingly, the allele rs6980093(A), associated with greater IFG activation during reading, forms a haplotype block with rs12533005(G), reported at risk in a rapid articulation task performed by normal readers (Peter et al., 2011). This block also includes allele rs2396753(G), reported in a risk haplotype for schizophrenia (Sanjuán et al., 2006). These observations are consistent and converge to the risk alleles combination rs12533005(G)/ rs2396753(G)/rs6980093(A). Additionally, rs6980093 is in high LD with one of the most reliable FOXP2 SNP, rs17137124, associated with phonological fluency and severity of degeneration of the inferior and dorsolateral frontal areas in elderly patients (Pado- 

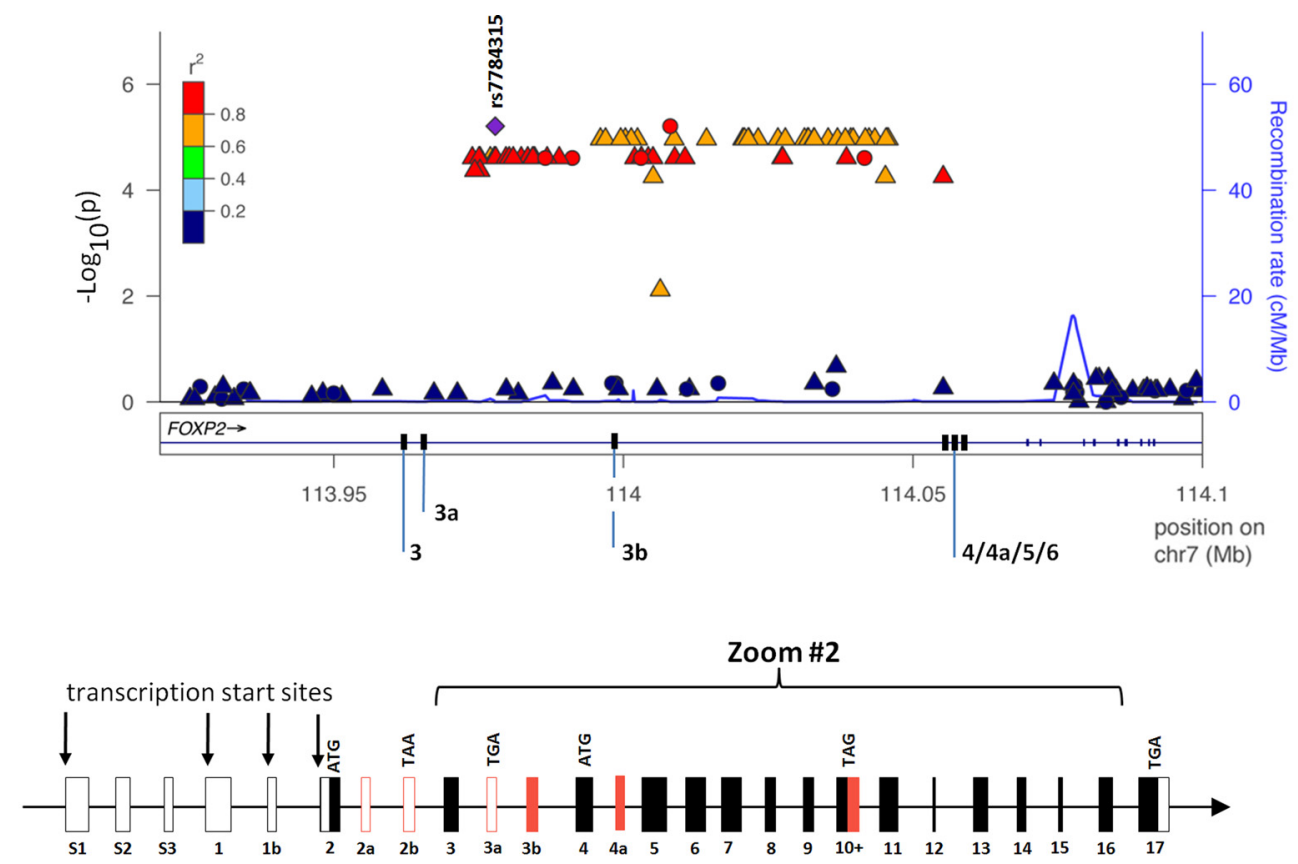

FOXP2 gene

Zoom \#1
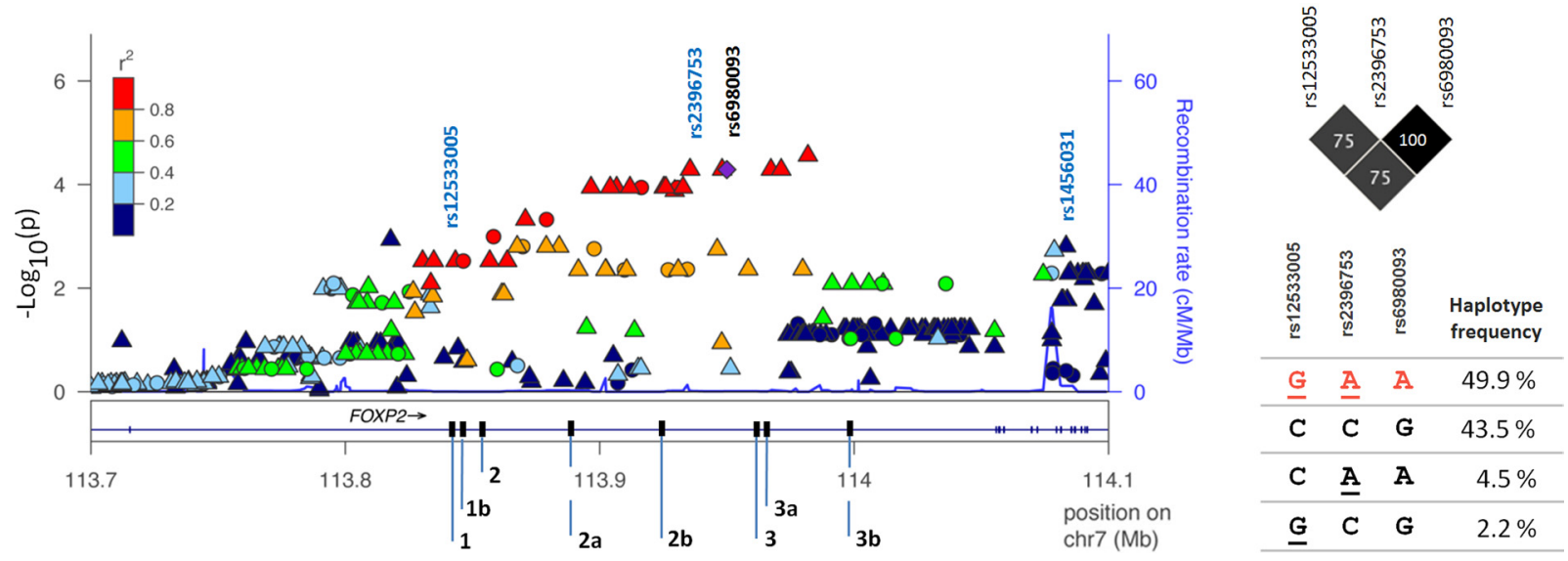

Figure 5. Zoomed view within the genetic regions of association with frontal activations (see annotations in Fig. 4). In the center are displayed the extents of the zoomed views relative to the exon structure of FOXP2. Full rectangles indicate translated exons and empty rectangles indicate untranslated exons. Red color indicates alternatively spliced exons. Arrows designate suggested transcription start sites. The two start codons (ATG) are indicated on exons 2 and 4, and stop codons (TAA, TGA, TAG) are indicated on alternatively splices exons $2 \mathrm{~b}, 3 \mathrm{a}, 10+$, and 17 (adapted from Schroeder and Myers, 2008). In the top is displayed the zoom on the FOXP2 locus associated with left precentral activity. Labels of exons covered by the locus are reported below. In the bottom is displayed the zoom on FOXP2 locus associated with left inferior frontal activity. We reported SNPs from the literature (blue label) that showed a trend of association with the cerebral phenotype $\left(\left(-\log _{10}(\boldsymbol{p})>2\right)\right.$ : rs 12533005 (Peter et al., 2010), rs2396753 (Sanjuán et al., 2006; Tolosa et al., 2010), and rs 1456031 (Sanjuán et al., 2006). Labels of exons covered by the locus are reported below. The table on the right shows the most frequent haplotypes corresponding to candidate SNPs in high LD with rs6980093. Underlined letters indicate alleles previously associated with a risk low articulation score for rs12533005 (Peter et al., 2010) and schizophrenia for rs2396753 (Sanjuán et al., 2006; Tolosa et al., 2010), and red letters indicate alleles associated with a higher IFG activation in the present study.

vani et al., 2010). This reinforces the conclusion that the development or operation of frontal language-related circuitry is altered by FOXP2 variants.

Contrary to the KIAA0319/TTRAP/THEM2 SNPs, no functional molecular mechanism has been proposed yet for the variants identified in FOXP2. However, they are in high LD with intronic SNPs covering a highly conserved nongenic region which comprised alternatively spliced exons (exons $3 \mathrm{a}$ and $3 \mathrm{~b}$ ) and lies in the vicinity of a putative promoter region (exons $1-1 \mathrm{~b})$. Alternative transcription start sites $1 / 1 \mathrm{~b}$ show greater cell- line specificity than those located in exons S1 and 2 (Schroeder and Myers, 2008) and produce alternative FOXP2 isoforms preferentially expressed, within the brain, in the thalamus (Sestan Lab Human Brain Atlas Microarrays), an important region for language and sensorimotor corticosubcortical loops (Haesler et al., 2004; Vargha-Khadem et al., 2005). Exon 3b adds 25 aa to the protein (Bruce and Margolis, 2002). These exons might allow the production of specific mRNA isoforms during development, possibly encoding different proteins, and thus putatively affect local subregions of language networks. Further studies are clearly 
needed to identify the functionally relevant SNPs in these FOXP2 haplotype blocks, and to study whether they impact on gene expression or protein production.

\section{Perspectives onto SLI and dyslexia}

SLI were primarily investigated as a deficit in oral language comprehension and production, while studies on dyslexia largely focused on the phonological deficit hypothesis. However, frequent cases of comorbidity between dyslexia and SLI (McArthur et al., 2000) raise the issue of whether these developmental disorders really reflect distinct cerebral abnormalities. Additionally, the nonhomogeneity of clinical groups may compromise the identification of cerebral and genetic markers associated with SLI or dyslexia. Considering these issues, Leonard and collaborators studied a pooled set of children characterized by deficits in oral language and reading and concluded that SLI and dyslexic subjects lie at the two extremities of the distribution of an anatomical index that characterizes brain volume and temporal asymmetry (Leonard et al., 2002, 2006). The present cortical doubledissociation of genetic determinants of SLI and dyslexia in typically developed individuals confirms their distinct contributions to the construction of the language network and supports the hypothesis that SLI and dyslexia may be, at least partially, distinct impairments (Bishop and Snowling, 2004), although the genetic and neuroimaging correlates of normal interindividual variability may be different from those underlying severe impairments.

\section{Limitations of our study}

We only explored a limited number of brain regions. It is possible that the variations of activation we observed are only secondary consequences of changes in other brain structures not imaged in our task, such as subcortical nuclei. For instance, voxel-based morphometry of affected K.E family members showed reduced gray matter density in IFG and left supplementary motor area but also in the cerebellum and the caudate nucleus (Watkins et al., 2002). Moreover, FOXP2 encodes a multidomain transcription factor that regulates various genetic pathways, and is expressed not only in the cortex, but also in thalamic nuclei and basal ganglia (Vargha-Khadem et al., 2005). Our correlational method does not exclude that the observed effects may be due to an indirect and possibly multiple causal chains, especially in adult subjects with a long brain development, education, and possible compensation for early deficits.

In conclusion, this study shows the feasibility of detecting genetic variants relevant for language disabilities from individual brain variations present in healthy populations. Using larger samples, this method could be expanded to wider segments of the genome, thus helping to identify other genes likely to contribute to normal and impaired language development. In particular, future studies should investigate genes whose expression is regulated by FOXP2 (Spiteri et al., 2007; Vernes et al., 2007; Konopka et al., 2009), such as CNTNAP2 (Vernes et al., 2008), and other candidate genes associated with dyslexia susceptibility $(D C D C 2$, ROBO1, DYX1C1).

\section{References}

Bartlett CW, Flax JF, Logue MW, Vieland VJ, Bassett AS, Tallal P, Brzustowicz LM (2002) A major susceptibility locus for specific language impairment is located on 13q21. Am J Hum Genet 71:45-55.

Benson RR, FitzGerald DB, LeSueur LL, Kennedy DN, Kwong KK, Buchbinder BR, Davis TL, Weisskoff RM, Talavage TM, Logan WJ, Cosgrove GR, Belliveau JW, Rosen BR (1999) Language dominance determined by whole brain functional MRI in patients with brain lesions. Neurology 52:798-809.
Bernal S, Dehaene-Lambertz G, Millotte S, Christophe A (2010) Two-yearolds compute syntactic structure on-line. Dev Sci 13:69-76.

Bishop DV, Snowling MJ (2004) Developmental dyslexia and specific language impairment: same or different? Psychol Bull 130:858-886.

Blau V, van Atteveldt N, Ekkebus M, Goebel R, Blomert L (2009) Reduced neural integration of letters and speech sounds links phonological and reading deficits in adult dyslexia. Curr Biol 19:503-508.

Brown S, Laird AR, Pfordresher PQ, Thelen SM, Turkeltaub P, Liotti M (2009) The somatotopy of speech: phonation and articulation in the human motor cortex. Brain Cogn 70:31-41.

Bruce HA, Margolis RL (2002) FOXP2: novel exons, splice variants, and CAG repeat length stability. Hum Genet 111:136-144.

Caylak E (2007) A review of association and linkage studies for genetical analyses of learning disorders. Am J Med Genet B Neuropsychiatr Genet 144B:923-943.

Cope N, Harold D, Hill G, Moskvina V, Stevenson J, Holmans P, Owen MJ, O'Donovan MC, Williams J (2005) Strong evidence that KIAA0319 on chromosome $6 \mathrm{p}$ is a susceptibility gene for developmental dyslexia. Am J Hum Genet 76:581-591.

De Fossé L, Hodge SM, Makris N, Kennedy DN, Caviness VS Jr, McGrath L, Steele S, Ziegler DA, Herbert MR, Frazier JA, Tager-Flusberg H, Harris GJ (2004) Language-association cortex asymmetry in autism and specific language impairment. Ann Neurol 56:757-766.

DeFries J (1996) Genetics of specific reading disability. Ment Retard Dev Disabil Res Rev 2:39-47.

Dehaene-Lambertz G, Dehaene S, Hertz-Pannier L (2002) Functional neuroimaging of speech perception in infants. Science 298:2013-2015.

Dennis MY, Paracchini S, Scerri TS, Prokunina-Olsson L, Knight JC, WadeMartins R, Coggill P, Beck S, Green ED, Monaco AP (2009) A common variant associated with dyslexia reduces expression of the KIAA0319 gene. PLoS Genet 5:e1000436.

Durbin RM, Abecasis GR, Altschuler DL, Auton A, Brooks LD, Gibbs RA, Hurles ME, McVean GA (2010) A map of human genome variation from population-scale sequencing. Nature 467:1061-1073.

Enard W, Przeworski M, Fisher SE, Lai CS, Wiebe V, Kitano T, Monaco AP, Pääbo S (2002) Molecular evolution of FOXP2, a gene involved in speech and language. Nature 418:869-872.

Fisher RH (1950) Statistical methods for research workers, Ed 11, pp $99-$ 101. London: Oliver and Boyd.

Fisher SE, Scharff C (2009) FOXP2 as a molecular window into speech and language. Trends Genet 25:166-177.

Fonteneaur E, van der Lely HK (2008) Electrical brain responses in languageimpaired children reveal grammar-specific deficits. PLoS One 3:e1832.

Francks C, Paracchini S, Smith SD, Richardson AJ, Scerri TS, Cardon LR, Marlow AJ, MacPhie IL, Walter J, Pennington BF, Fisher SE, Olson RK, DeFries JC, Stein JF, Monaco AP (2004) A 77-kilobase region of chromosome 6p22.2 is associated with dyslexia in families from the United Kingdom and from the United States. Am J Hum Genet 75:1046-1058.

Galaburda AM, Sherman GF, Rosen GD, Aboitiz F, Geschwind N (1985) Developmental dyslexia: four consecutive patients with cortical anomalies. Ann Neurol 18:222-233.

Galaburda AM, LoTurco J, Ramus F, Fitch RH, Rosen GD (2006) From genes to behavior in developmental dyslexia. Nat Neurosci 9:1213-1217.

Gao X, Starmer J, Martin ER (2008) A multiple testing correction method for genetic association studies using correlated single nucleotide polymorphisms. Genet Epidemiol 32:361-369.

Gao X, Becker LC, Becker DM, Starmer JD, Province MA (2010) Avoiding the high Bonferroni penalty in genome-wide association studies. Genet Epidemiol 34:100-105.

Gauger LM, Lombardino LJ, Leonard CM (1997) Brain morphology in children with specific language impairment. J Speech Lang Hear Res 40:1272-1284.

Haesler S, Wada K, Nshdejan A, Morrisey EE, Lints T, Jarvis ED, Scharff C (2004) FoxP2 expression in avian vocal learners and non-learners. J Neurosci 24:3164-3175.

Harold D, Paracchini S, Scerri T, Dennis M, Cope N, Hill G, Moskvina V, Walter J, Richardson AJ, Owen MJ, Stein JF, Green ED, O'Donovan MC, Williams J, Monaco AP (2006) Further evidence that the KIAA0319 gene confers susceptibility to developmental dyslexia. Mol Psychiatry 11:1085-1091.

Jentschke S, Koelsch S, Sallat S, Friederici AD (2008) Children with specific 
language impairment also show impairment of music-syntactic processing. J Cogn Neurosci 20:1940-1951.

Kang HJ, Kawasawa YI, Cheng F, Zhu Y, Xu X, Li M, Sousa AM, Pletikos M, Meyer KA, Sedmak G, Guennel T, Shin Y, Johnson MB, Krisnik Z, Mayer S, Fertuzinhos S, Umlauf S, Lisgo SN, Vortmeyer A, Weinberger DR, et al. (2011) Spatio-temporal transcriptome of the human brain. Nature 478: 483-489.

Konopka G, Bomar JM, Winden K, Coppola G, Jonsson ZO, Gao F, Peng S, Preuss TM, Wohlschlegel JA, Geschwind DH (2009) Human-specific transcriptional regulation of CNS development genes by FOXP2. Nature 462:213-217.

Lai CS, Fisher SE, Hurst JA, Vargha-Khadem F, Monaco AP (2001) A forkhead-domain gene is mutated in a severe speech and language disorder. Nature 413:519-523.

Leonard CM, Eckert MA, Lombardino LJ, Oakland T, Kranzler J, Mohr CM, King WM, Freeman A (2001) Anatomical risk factors for phonological dyslexia. Cereb Cortex 11:148-157.

Leonard CM, Lombardino LJ, Walsh K, Eckert MA, Mockler JL, Rowe LA, Williams S, DeBose CB (2002) Anatomical risk factors that distinguish dyslexia from SLI predict reading skill in normal children. J Commun Disord 35:501-531.

Leonard C, Eckert M, Given B, Virginia B, Eden G (2006) Individual differences in anatomy predict reading and oral language impairments in children. Brain 129:3329-3342.

Li Y, Willer CJ, Ding J, Scheet P, Abecasis GR (2010) MaCH: using sequence and genotype data to estimate haplotypes and unobserved genotypes. Genet Epidemiol 34:816-834.

Liégeois F, Baldeweg T, Connelly A, Gadian DG, Mishkin M, Vargha-Khadem F (2003) Language fMRI abnormalities associated with FOXP2 gene mutation. Nat Neurosci 6:1230-1237.

Luciano M, Lind PA, Duffy DL, Castles A, Wright MJ, Montgomery GW, Martin NG, Bates TC (2007) A haplotype spanning KIAA0319 and TTRAP is associated with normal variation in reading and spelling ability. Biol Psychiatry 62:811-817.

MacDermot KD, Bonora E, Sykes N, Coupe AM, Lai CS, Vernes SC, VarghaKhadem F, McKenzie F, Smith RL, Monaco AP, Fisher SE (2005) Identification of FOXP2 truncation as a novel cause of developmental speech and language deficits. Am J Hum Genet 76:1074-1080.

Maisog JM, Einbinder ER, Flowers DL, Turkeltaub PE, Eden GF (2008) A meta-analysis of functional neuroimaging studies of dyslexia. Ann N Y Acad Sci 1145:237-259.

Maldjian JA, Laurienti PJ, Kraft RA, Burdette JH (2003) An automated method for neuroanatomic and cytoarchitectonic atlas-based interrogation of fMRI data sets. Neuroimage 19:1233-1239.

McArthur GM, Hogben JH, Edwards VT, Heath SM, Mengler ED (2000) On the "specifics" of specific reading disability and specific language impairment. J Child Psychol Psychiatry 41:869-874.

Newbury DF, Paracchini S, Scerri TS, Winchester L, Addis L, Richardson AJ, Walter J, Stein JF, Talcott JB, Monaco AP (2011) Investigation of dyslexia and SLI risk variants in reading- and language-impaired subjects. Behav Genet 41:90-104.

Padovani A, Cosseddu M, Premi E, Archetti S, Papetti A, Agosti C, Bigni B, Cerini C, Paghera B, Bellelli G, Borroni B (2010) The speech and language FOXP2 gene modulates the phenotype of frontotemporal lobar degeneration. J Alzheimers Dis 22:923-931.

Paracchini S, Thomas A, Castro S, Lai C, Paramasivam M, Wang Y, Keating BJ, Taylor JM, Hacking DF, Scerri T, Francks C, Richardson AJ, WadeMartins R, Stein JF, Knight JC, Copp AJ, Loturco J, Monaco AP (2006) The chromosome 6p22 haplotype associated with dyslexia reduces the expression of KIAA0319, a novel gene involved in neuronal migration. Hum Mol Genet 15:1659-1666.

Paracchini S, Steer CD, Buckingham LL, Morris AP, Ring S, Scerri T, Stein J, Pembrey ME, Ragoussis J, Golding J, Monaco AP (2008) Association of the KIAA0319 dyslexia susceptibility gene with reading skills in the general population. Am J Psychiatry 165:1576-1584.

Paulesu E, Démonet JF, Fazio F, McCrory E, Chanoine V, Brunswick N, Cappa SF, Cossu G, Habib M, Frith CD, Frith U (2001) Dyslexia: cultural diversity and biological unity. Science 291:2165-2167.

Pennington BF, Gilger JW, Pauls D, Smith SA, Smith SD, DeFries JC (1991) Evidence for major gene transmission of developmental dyslexia. JAMA 266:1527-1534.
Peter B, Raskind WH, Matsushita M, Lisowski M, Vu T, Berninger VW, Wijsman EM, Brkanac Z (2011) Replication of CNTNAP2 association with nonword repetition and support for FOXP2 association with timed reading and motor activities in a dyslexia family sample. J Neurodev Disord 3:39-49.

Pinel P, Dehaene S (2010) Beyond hemispheric dominance: brain regions underlying the joint lateralization of language and arithmetic to the left hemisphere. J Cogn Neurosci 22:48-66.

Pinel P, Thirion B, Meriaux S, Jobert A, Serres J, Le Bihan D, Poline JB, Dehaene S (2007) Fast reproducible identification and large-scale databasing of individual functional cognitive networks. BMC Neurosci 8:91.

Pruim RJ, Welch RP, Sanna S, Teslovich TM, Chines PS, Gliedt TP, Boehnke M, Abecasis GR, Willer CJ (2010) LocusZoom: regional visualization of genome-wide association scan results. Bioinformatics 26:2336-2337.

Rice ML, Smith SD, Gayán J (2009) Convergent genetic linkage and associations to language, speech and reading measures in families of probands with Specific Language Impairment. J Neurodev Disord 1:264-282.

Sanjuán J, Tolosa A, González JC, Aguilar EJ, Pérez-Tur J, Nájera C, Moltó MD, de Frutos R (2006) Association between FOXP2 polymorphisms and schizophrenia with auditory hallucinations. Psychiatr Genet 16:67-72.

Schroeder DI, Myers RM (2008) Multiple transcription start sites for FOXP2 with varying cellular specificities. Gene 413:42-48.

Shaywitz SE, Escobar MD, Shaywitz BA, Fletcher JM, Makuch R (1992) Evidence that dyslexia may represent the lower tail of a normal distribution of reading ability. N Engl J Med 326:145-150.

Shu W, Cho JY, Jiang Y, Zhang M, Weisz D, Elder GA, Schmeidler J, De Gasperi R, Sosa MA, Rabidou D, Santucci AC, Perl D, Morrisey E, Buxbaum JD (2005) Altered ultrasonic vocalization in mice with a disruption in the Foxp2 gene. Proc Natl Acad Sci U S A 102:9643-9648.

Silani G, Frith U, Demonet JF, Fazio F, Perani D, Price C, Frith CD, Paulesu E (2005) Brain abnormalities underlying altered activation in dyslexia: a voxel based morphometry study. Brain 128:2453-2461.

SLIC (2004) Highly significant linkage to the SLI1 locus in an expanded sample of individuals affected by specific language impairment. Am J Hum Genet 74:1225-1238.

Spiteri E, Konopka G, Coppola G, Bomar J, Oldham M, Ou J, Vernes SC, Fisher SE, Ren B, Geschwind DH (2007) Identification of the transcriptional targets of FOXP2, a gene linked to speech and language, in developing human brain. Am J Hum Genet 81:1144-1157.

Stromswold K (2001) The heritability of language: A review and metaanalysis of twin, adoption, and linkage studies. Language 77:647-723.

Tolosa A, Sanjuán J, Dagnall AM, Moltó MD, Herrero N, de Frutos R (2010) FOXP2 gene and language impairment in schizophrenia: association and epigenetic studies. BMC Med Genet 11:114.

Vargha-Khadem F, Watkins K, Alcock K, Fletcher P, Passingham R (1995) Praxic and nonverbal cognitive deficits in a large family with a genetically transmitted speech and language disorder. Proc Natl Acad Sci U S A 92:930-933.

Vargha-Khadem F, Watkins KE, Price CJ, Ashburner J, Alcock KJ, Connelly A, Frackowiak RS, Friston KJ, Pembrey ME, Mishkin M, Gadian DG, Passingham RE (1998) Neural basis of an inherited speech and language disorder. Proc Natl Acad Sci U S A 95:12695-12700.

Vargha-Khadem F, Gadian DG, Copp A, Mishkin M (2005) FOXP2 and the neuroanatomy of speech and language. Nat Rev Neurosci 6:131-138.

Vernes SC, Spiteri E, Nicod J, Groszer M, Taylor JM, Davies KE, Geschwind DH, Fisher SE (2007) High-throughput analysis of promoter occupancy reveals direct neural targets of FOXP2, a gene mutated in speech and language disorders. Am J Hum Genet 81:1232-1250.

Vernes SC, Newbury DF, Abrahams BS, Winchester L, Nicod J, Groszer M, Alarcón M, Oliver PL, Davies KE, Geschwind DH, Monaco AP, Fisher SE (2008) A functional genetic link between distinct developmental language disorders. N Engl J Med 359:2337-2345.

Watkins KE, Vargha-Khadem F, Ashburner J, Passingham RE, Connelly A, Friston KJ, Frackowiak RS, Mishkin M, Gadian DG (2002) MRI analysis of an inherited speech and language disorder: structural brain abnormalities. Brain 125:465-478.

Zhao Y, Ma H, Wang Y, Gao H, Xi C, Hua T, Qiu G (2010) Association between FOXP2 gene and speech sound disorder in Chinese population. Psychiatry Clin Neurosci 64:565-573. 